\title{
Thermochromism in Oligothiophenes: The Role of the Internal Rotation
}

\author{
Nuran Elmacı \\ Izmir Institute of Technology, Department of Chemistry, Gülbahçeköyü, Urla, İmir 35437, Turkey
}

\section{Ersin Yurtsever*}

Koç University, Department of Chemistry, Rumeli Feneri Yolu, Sartyer Istanbul 34450, Turkey

Received: August 15, 2002; In Final Form: October 16, 2002

\begin{abstract}
The relation between thermochromism in polythiophenes and the internal rotation is studied by densityfunctional theory (DFT). For head-to-tail methyl-substituted thiophenes, rotational potential maps of dimer and trimer are calculated. With time-dependent-DFT methodology the absorption spectra of these oligomers in UV-vis region are calculated as functions of the torsional angles. Theoretical temperature-dependent UVvis spectra are generated from the distribution of torsional angles. The results show that the internal rotation alone is not sufficient to explain the mechanism of thermochromism.
\end{abstract}

\section{Introduction}

It has been extensively reported that substituted polythiophenes and oligothiophenes display thermochromic and solvatochromic optical properties. ${ }^{1}$ These novel polymers are very important for the production of nonlinear optical materials. ${ }^{2-3}$

It is generally claimed that, the chromic behaviors are related to a conformational change in the planar form of the backbone. Deviation from planarity decreases the conjugation length that then results in shifts in the UV-vis absorption spectrum. ${ }^{1}$ In the case of thermochromism, a blue shift is observed as the temperature is increased. In a study of the nonlinear optical behavior of conjugated polymers, thermochromic behavior of several substituted polythiophenes is reported. Poly(3- alkylthiophene), poly(3-(alkylthio)thiophene), and poly (3-alkoxy4-methylthiophene) have shown thermochromism in that series. ${ }^{4}$ A conformational analysis along the twisting of the backbone was used to explain thermochromism of those polymers. It was suggested that those thermochromic polymers can exist in the coplanar and nearly planar conformations at low temperatures but they can form configurations with twisted backbone as a result of disordering of the side chains at high temperatures. ${ }^{5,6}$ Poly(3,3'-dialkyl-2,2'-bithiophene) and poly(3,3'-dialkoxy-2,2'bithiophene) were not thermochromic as the coplanar conformations are unfavored due to the presence of large steric hindrances for the former polymer. The later polymer can preserve its coplanar arrangements even at high temperatures.

Similar to the polythiophenes, polyphenylenes and polyfluorenes derivatives ( $\pi$-conjugated polymers) possess thermochromic and solvatochromic properties. For polyphenylenes and polyfluorenes, it was reported that, the derivatives, which possess thermochromism, had rather flexible backbones with rather small energy barriers against planarity. On the other hand, nonchromic derivatives had either favored planar conformations even at high temperatures or large energy barriers between coplanar and twisted conformations. ${ }^{7}$ Not only $\pi$-conjugated polymers have the chromic behavior but also $\sigma$-conjugated polymers may show similar behavior. It was pointed out that the driving forces for the thermochromism and solvatochromism are the same for some nonionic polar polysilanes due to entanglement/disentanglement of the main chain by polar/ nonpolar solvent or strong hydrogen bond formation or by heating 8

Some of the polysilane derivatives have a continuous shift in their spectra and some others have a discontinuous shift. Sanji et al. used a statistical model for the cooperative thermochromic transition of polysilanes. ${ }^{9}$ They computed the partition function of a single polysilane chain as a sum of nearest neighbor interactions, trans and two gauche $(+,-)$ conformations. A fit was made to experimental spectra to estimate some parameters: stabilization energies and entropies. Three different categories were suggested for the thermochromic behavior of polysilanes depending on the nature of the alkyl group: transition type-intermediate and nontransition. If the stabilization energy between two ordered states (trans and gauche) is large, they are classified as a transition type (discontinuous shift) thermochromism.

The thermochromic polythiophenes can also possess either a continuous blue shift ${ }^{10}$ or an isobestic point (discontinuous) ${ }^{11}$ depending on the linkage of the thiophene units (head to tail (HT), tail to tail (TT), or head to head (HH)). The existence of isobestic point was explained by the formation of twistons (conformational changes on the backbone upon heating). Yang et al. proposed a model ${ }^{12}$ for the prediction of thermochromism of regioregular and nonregioregular poly (3-alkylthiophenes) using the polymers, which have different head to tail content. (Polymers with $80 \%$ HT and $100 \%$ HT (polymers with long alkyl chain) content have an isobestic point on the other hand $>90 \%$ HT content have a continuous blue shift.) The effects of the side chain length and regioregularity on the thermochromism was summarized as polymers with moderate HT content shows thermochromism with an isobestic point independent of the size of alkyl group while HT regioregular polymers with short side chains exhibit a continuous shift upon heating, the one with longer side chains has an isobestic point. Two mechanisms were suggested; a direct transition from crystalline state to the 
disordered state (isobestic point) or a transition from ordered to quasi-ordered state that has some twisted chains then to disordered state (continuous shift).

Zerbi et al. has predicted two phases (ordered or disordered) at room temperature from the analysis of IR and Raman spectra of polyalkylthiophenes. ${ }^{13}$ In the ordered phase, the alkyl chains have trans-planar structure and the main chains are in quasiplanar or coplanar structures whereas in the disordered phase, the alkyl groups are coiled and the backbone is twisted. Thus, increasing the temperature favors disordered phase.

The conformational analysis of terthiophene, methyl, methoxy and ethyl substituted terthiophenes were carried out by DiCesare et al. using ab initio calculations at the HF/3-21G* level. ${ }^{14}$ It is shown that, the most stable conformation of unsubstituted terthiophene is located at the twist angle of $147^{\circ}$, and the maxima are located at $0^{\circ}, 90^{\circ}$, and $180^{\circ}$ (the origin of the torsional angle, $\varphi=0$ corresponds to cis rotamer). For the methoxy terthiophene the planar conformation is the most favored one with a larger energy barrier at $90^{\circ}$. Our former semiempirical calculations have also shown that the methylbithiophene preferred planar cis-type structures. ${ }^{15}$ The more twisted structures are favored with the introduction of methyl and ethyl side groups because of the steric effect. Their absorption and fluorescence spectra were also compared by DiCesare et al. ${ }^{16}$ The absorption spectra of methoxy substituted is red-shifted, more structured, sharper compared to the unsubstituted one, whereas alkyl substituted ones are blue-shifted, unstructured, and broad. They concluded that all the molecules become more planar in their first excited-state according to their fluorescence spectra.

The oligothiophenes exhibit also chromic behavior similar to their corresponding polymers. The thermochromism of terthiophenes and quaterthiophenes has been reported by Cesare et al. in a series of papers. ${ }^{16-18}$ The thermochromism in substituted terthiophenes is coupled with the conformational changes in contrast to the unsubstituted terthiophene which shows an important excitonic effect.

The blue shift upon heating may also due to the $\pi$-stacking. ${ }^{19}$ The study of stacking of regioregular poly-3-hexylthiophenes showed that the polymer exhibits the photoinduced polarizability in all directions; the backbone direction as well as the two others. It was also found that the thermochromic transition temperature for poly(3-alkylthiophene) thin films decreases with increasing the length of the alkyl-side groups. ${ }^{20}$ The thermochromic behavior was joined with the ordered-disordered transitions of side groups due to the results of the temperature-dependent IR measurements.

There are several lattice simulation studies on the conformations of the side chain and the backbone of poly (3alkylthiophene)s. ${ }^{21-23,25}$ Some of them showed that the geometry changes other than the twisting of the backbone might have also caused the blue shift upon heating. Besides the interring rotations, the booklike folding of the rings was also predicted in those studies. The cis and gauche conformations of the side chains were predicted as the low energy structures. If the main chain is distorted by either twisting or folding, the alkyl chains took nontrans conformations and that was explained such that, the thermal energy was stored in the side chains and transformed to the polymer skeleton, which then broke the stiffness of the backbone and decreased the $\pi$-conjugation length. ${ }^{21,22}$

In other atomistic lattice simulations, the effect of the temperature on the structural change of the poly(3-butyl thiophene) backbone upon was investigated. ${ }^{23}$ The lattices with different sequences of interring torsions that preserve periodicity of the main chain were taken into account. In that work thermochromism is related by the existence of alternative chains along the stacking axis $b$, which shift by $c / 2$ along $c$ to produce eclipsed-ring structured chains that can easily twist upon heating.

An X-ray study showed that the length of alkyl groups on the poly(3-alkylthiophenes) plays an important role on the molecular chain packing. ${ }^{24}$ Longer alkyl sides lead to lower melting point, higher $d$ spacing between layers, and higher order in the side chains.

The role of alkyl chains on the thermochromism of poly(3alkylthiophene)s was also studied using lattice and molecular simulation dynamics methods. ${ }^{25}$ It was predicted that the side chains have a tendency to tilt. The side chains may be either coplanar with the backbone or tilted as a result of packing effect (depending on the preparation conditions). The dissipation of the steric energy stored in the side chains to the backbone leads to twisting of the main chain. However for the octyl substituted polymer that is not thermochromic, the steric energy is shared by the side chain and the main chain and so polymer has a twist backbone even at low temperatures. The proposed mechanism in that work for the thermochromism is the twisting of the main chain as a result of the transmission of the energy from the side chains to the main chain.

In most of the above work, thermochromism is explained by the rotation of the backbone; however, the individual effects of such conformational changes occurring in single polymer chains or in bulk system cannot be distinguished. The role of $\pi$-stacking, which can be related to the bulk effect on thermochromism, is also not clearly understood.

In this work we present accurate density-functionaltheory (DFT) calculations along the rotational motion of unsubstituted and methyl-substituted bithiophenes and terthiophenes. UV-vis spectrum at each geometry is calculated; these excitations are then combined via Boltzmann probabilities to compute temperature-dependent UV-vis spectra of single oligomers.

\section{Results and Discussion}

Quantum mechanical calculations have been performed with GAUSSIAN98. ${ }^{26}$ The rotational maps for the ground state have been obtained within DFT using the three-parameter hybrid functional of Becke. ${ }^{27}$ The 6-31g(d) basis set is used, and all internal coordinates are optimized except for the torsional angle to be studied. For the methyl-substituted thiophenes, we have only used to regioregular head-to-tail (HT) arrangement of the alkyl groups.

Rotational Energy Profiles. There has been numereous reports on the torsional potentials of bithiophenes. It has been pointed out that the correct behavior of the potential cannot be obtained without correlation effects. In fact, MP2 type approached differ to a great extent from DFT results. ${ }^{28}$ On the other hand, it was also reported that DFT geometry and energetics were comparable to experimental data especially for the B3LYP functionals. ${ }^{29}$

In Figure 1 we present the rotational potential energy profiles for bithiophene (2T) and HT 3,3'-dimethyl-2,2'-bithiophene (methyl-2T). The rotational angle is defined so that $\varphi=180^{\circ}$ corresponds to anticonfirmation of two rings. The rotational motion in $2 \mathrm{~T}$ and methyl-2T display the same characteristics that there are two minimum energy conformations (syngauche and anti-gauche) very close to each other in energy. Our energy values (for 2T) agree with the calculations of Karpfen ${ }^{28}$ but differ from those found by Bongini (for methyl-2T). ${ }^{29}$ The 


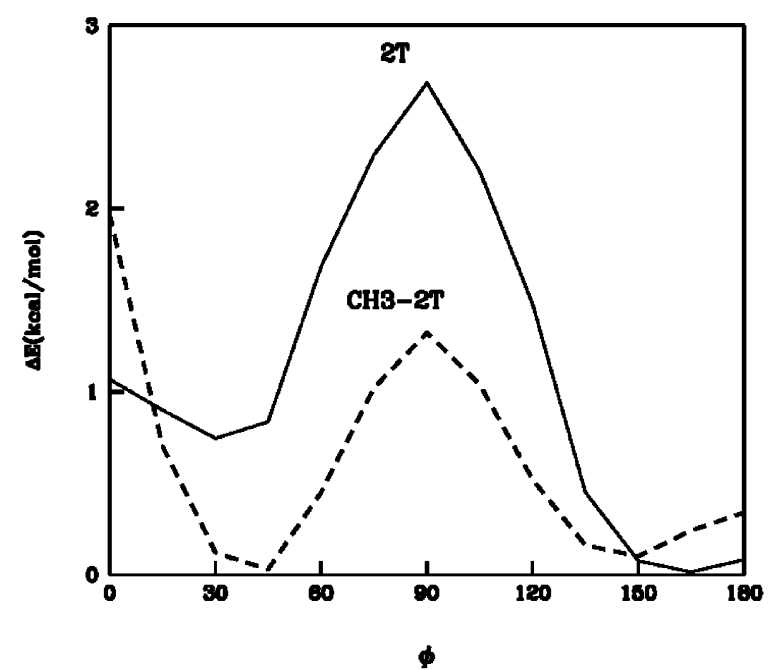

Figure 1. Rotational potential energy profiles for $2 \mathrm{~T}$ and methyl-2T.

latter results may correspond to local minima since the absolute energies reported by them are quite above of ours ${ }^{29}$ For example, they have reported the B3LYP energy as (hartree) -1183.2201 for $\varphi=180$, and we have calculated it to be -1183.45185 .

Rotational barriers for $2 \mathrm{~T}$ and methyl-2T are found to be 2.8 and $1.3 \mathrm{kcal} / \mathrm{mol}$, respectively. In case of $2 \mathrm{~T}$, the global minimum is at $157^{\circ}$ and syn-gauche local minimum is around $30^{\circ}$ and $0.8 \mathrm{kcal} /$ above the global minimum. For methyl-2T, two minima lie very close in energy with the global minimum being at $40^{\circ}$.

Excited-State Calculations. The UV-vis spectra of 2T, and those of methyl-2T, are calculated by the time-dependentdensity-functional-theory (TDDFT) ${ }^{30}$ with the basis set 6-31g(d,p). We have chosen this method as it was reported that TDDFT results agree well with the experimental results for the excitation spectra of terthiophene and terthiophene-S,S-dioxide. ${ }^{31}$

The structures of dimers are taken from the optimized structures with B3LYP/6-31g(d). From TDDFT, three lowest lying $\mathrm{S}$-states are calculated. The lowest state $\mathrm{S}_{1}$ has always the largest transition probability, $S_{2}$ is almost invariably forbidden, and $S_{3}$ has a very weak intensity. The excitation energy as well as the intensity is reported in Figure 2 a for $S_{1}$ and in Figure $2 b$ for $S_{3}$ as functions of the rotational angle.

The calculations for $2 \mathrm{~T}$ and methyl-2T are very similar in all aspects. In fact, another series of calculations on ethyl-2T is carried out but is not reported, as it does not offer any significantly different behavior. The energy of the strongest absorption to $S_{1}$ increases as the bithiophene twists out of planar structure; however, at the same time the intensity decreases, and at $\varphi=90$, it becomes a forbidden transition. Using the lowest three excitations we plot the UV-vis spectra at different rotational structures for methyl-2T in Figure 3. Each excitation is expressed as a weighted Gaussian centered at the excitation energy with a half-bandwidth of $0.5 \mathrm{eV}$, weights are directly proportional to the oscillator strengths. ${ }^{32}$

The absorption spectra for $0<\varphi<90$ are very similar to those for $90<\varphi<180$; i.e., the maxima for $\varphi=75$ and $\varphi=$ 105 differ by only $0.02 \mathrm{eV}$ and the intensities are almost the same. In order for the clarity of presentation, only the first group is displayed. There is a strong blue shift in the absorptions as the dimer rotates around the monomer-monomer bond. This shift forms the basis of the belief that thermochromism is mostly due to the variations on the torsional angle.
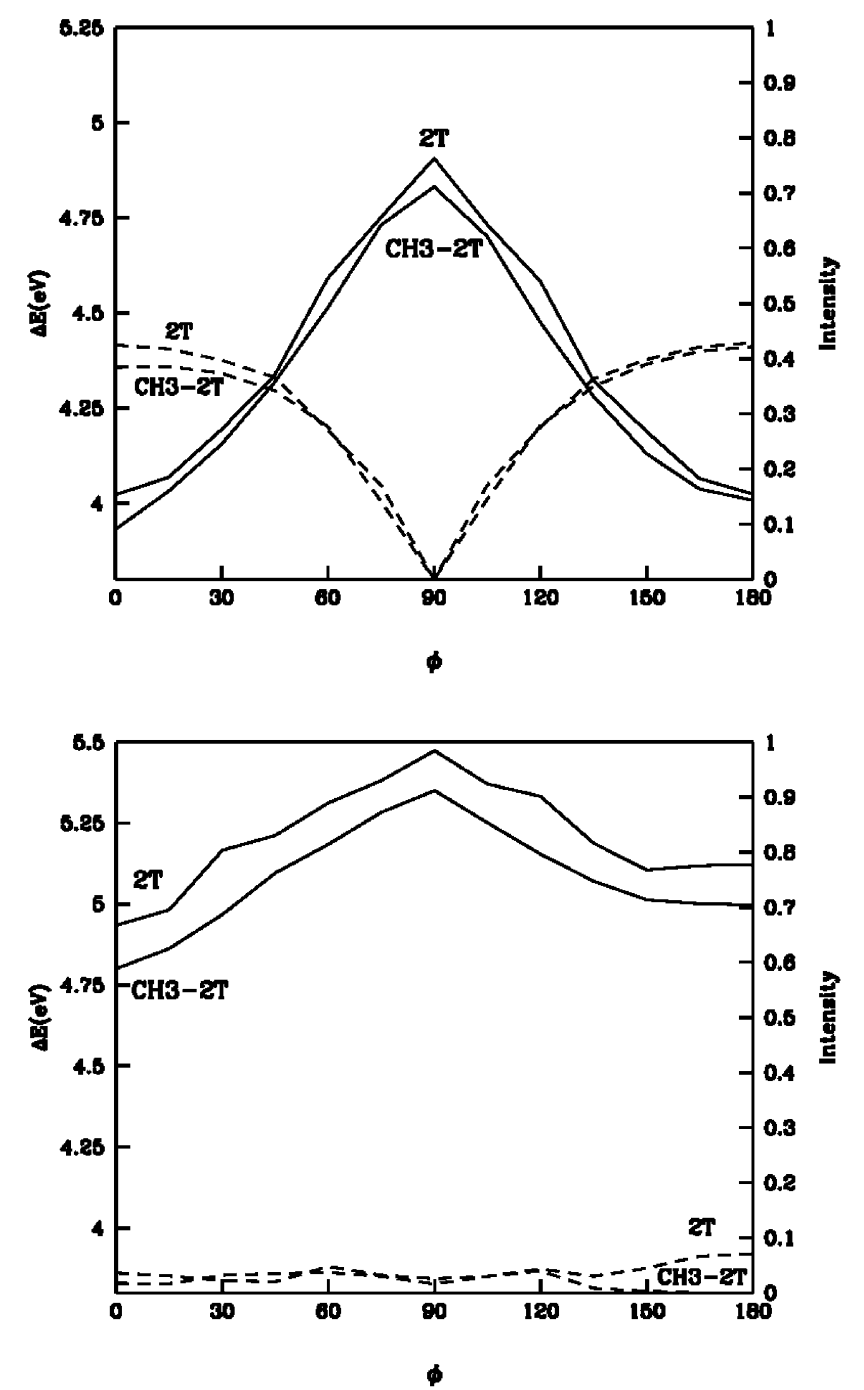

Figure 2. Excitation energy and the intensity of absorption for $2 \mathrm{~T}$ and $\mathrm{CH} 3-2 \mathrm{~T}$. (Continuous lines are for the energy, dotted lines are for the intensity). (a) $S_{1}$. (b) $S_{3}$.

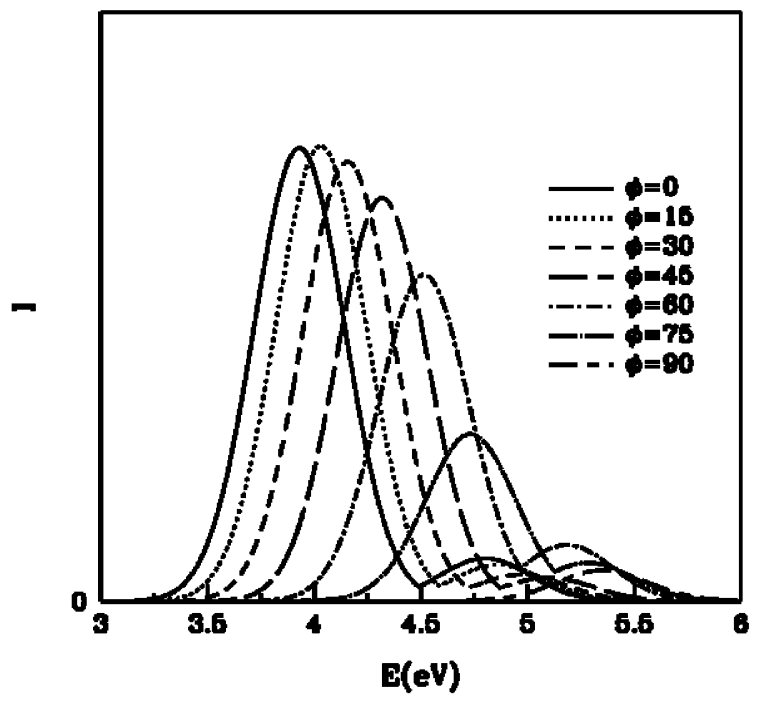

Figure 3. UV - Vis spectra of methyl-2T as a function of the torsional angle.

Temperature-Dependent Absorption Spectra. However, a better approach should involve the relative populations of all states of the arising from the rotational motion. We proceed to 


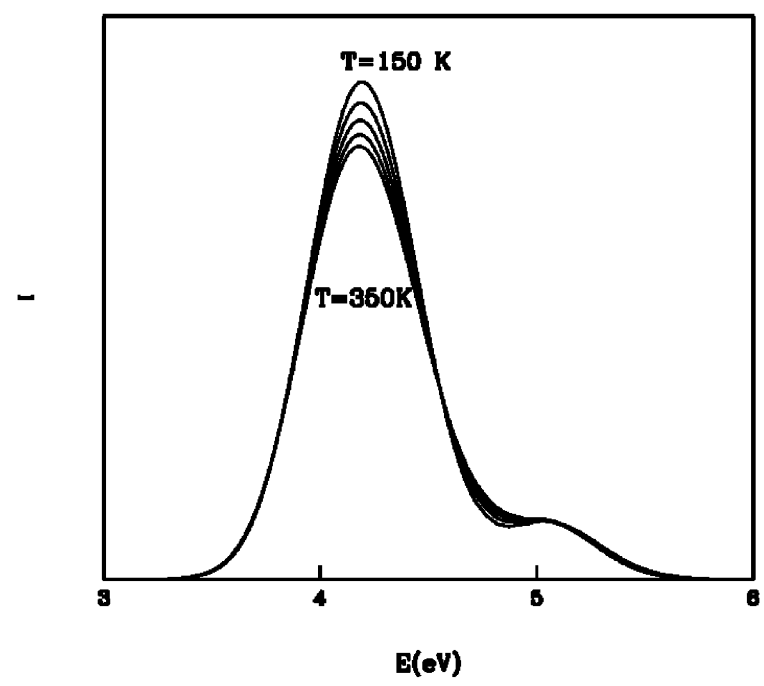

Figure 4. Temperature-dependent absorption spectra of methyl-2T.

define a Boltzmann probability in terms of the rotational angle $\varphi$ :

$$
P(\varphi, T)=\mathrm{e}^{\left(E(\varphi)-E_{0}\right) / k T}
$$

where $E_{0}$ is the lowest energy of the rotational potential energy function. The temperature dependent absorption can then simply be written as

$$
\Psi(T)=\int \Psi(\varphi) P(\varphi, T) \mathrm{d} \varphi
$$

Here $\Psi(\varphi)$ defines the spectrum of the dimer at a given angle $\varphi$. Our spectra and probability functions are all discrete variables so the integral in eq 2 is replaced by a summation. In Figure 4 we present the temperature-dependent spectra for methyl-2T. We clearly do not observe any reasonable shift in the absorption peaks. In fact, a simple look at the distribution of the rotational angles as a function of the temperature shows that these populations do not change significantly with temperature. These results show that thermochromism is not a result of the rotational motion, at least for the dimers of thiophene and methylthiophene. However, to be able to conclude that internal rotation is not the basic source of thermochromism, there are two more questions to be answered. First of all, the length of the alkyl group may play an important role in thermochromism. To clarify this issue we have calculated the spectra for ethyl-2T as a function of the rotational angle and a blue shift similar in magnitude to that of Figure 3 is observed. On the other hand, the rotational barriers in alkyl thiophenes up to pentyl-2T (unpublished results) again are in the same order as in methyl$2 \mathrm{~T}$. Hence we do not expect any different behavior for alkyl thiophene dimers with longer substitutions. The second possibility is such that the temperature-dependent behavior is not observed in dimers but it may appear for longer chains.

To test this hypothesis, we have calculated the full rotational map for the methyl-terthiophene (methyl-3T) again with 6-31g(d) basis set and the B3LYP functional (Figure 5). The global mimimum is found to be at the location $\left(45^{\circ}, 315^{\circ}\right)$. The maxima lie at $\left(90^{\circ}, 90^{\circ}\right),\left(90^{\circ}, 70^{\circ}\right),\left(270^{\circ}, 90^{\circ}\right)$, and $\left(270^{\circ}, 270^{\circ}\right)$. The energy of these structures is $2.9 \mathrm{kcal} / \mathrm{mol}$ above the minimum energy conformation $\left(0^{\circ}, 0^{\circ}\right)$ conformation corresponds to ciscis structure).

TDDFT calculations are performed over a grid with intervals of $45^{\circ}$. The lowest three excitations are recorded. Two of these excitations are either forbidden or have extremely low intensities. In Figures 6 and 7, the energy of the lowest and the strongest excitation and its intensity are presented as functions of two torsional angles. As in the dimer case, the excitation energy increases as the torsional angle changes the structure from planar to perpendicular form. At the same time, the intensity decreases. For example, at $\left(90^{\circ}, 90^{\circ}\right)$ this transition becomes forbidden.

To calculate the temperature-dependent absorption spectra, a Boltzmann averaging over two torsional angles is performed:

$$
\Psi(\mathrm{T})=\int \Psi\left(\varphi_{1}, \varphi_{2}\right) P\left(\varphi_{1}, \varphi_{2}, T\right) \mathrm{d} \varphi_{1} \mathrm{~d} \varphi_{2}
$$

The results are presented in Figure 8.

The maximum has shifted to a much lower energy (from 4.3 to $3.5 \mathrm{eV}$ ) from dimer to trimer. It is well-known fact that in oligothiophenes, the energy for the lowest absorption decreases with the length of the oligomer. ${ }^{33}$ However, similar to dimers, the temperature dependence is only observed in the intensity of the spectra. In the temperature range of 250-350 K, there is no detectable change in the maxima of absorption.

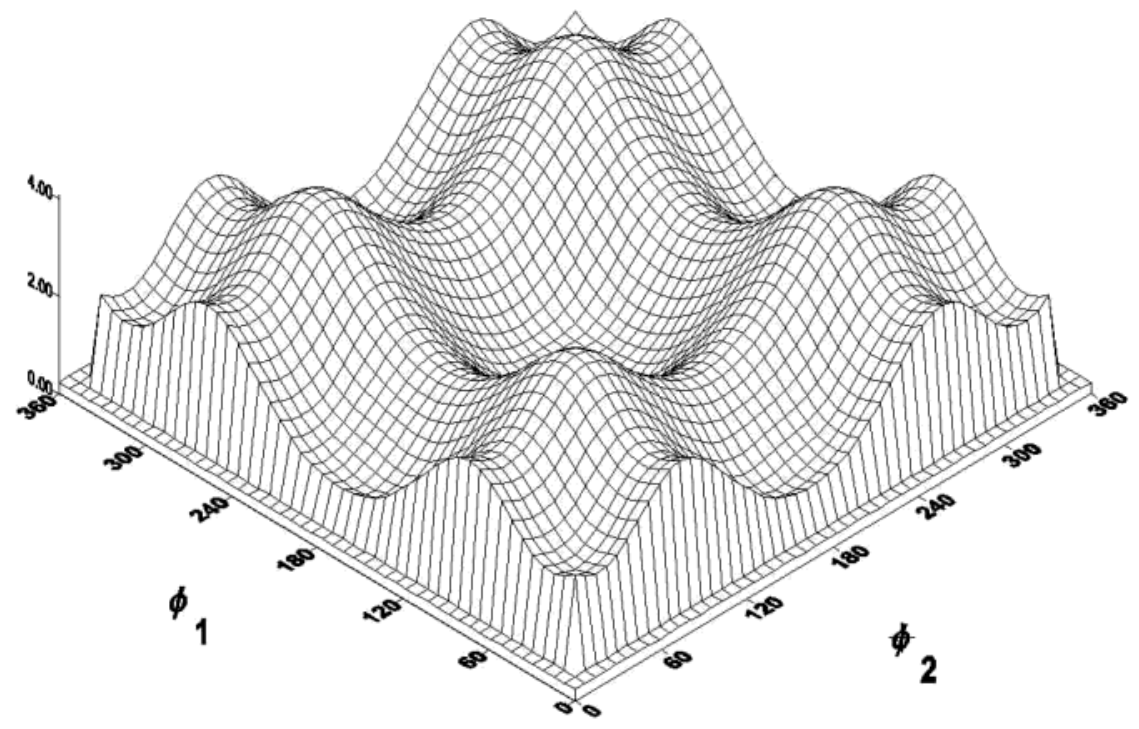

Figure 5. Two-dimensional rotational map for methyl-3T (in $\mathrm{kcal} / \mathrm{mol}$ with respect to minimum energy). 


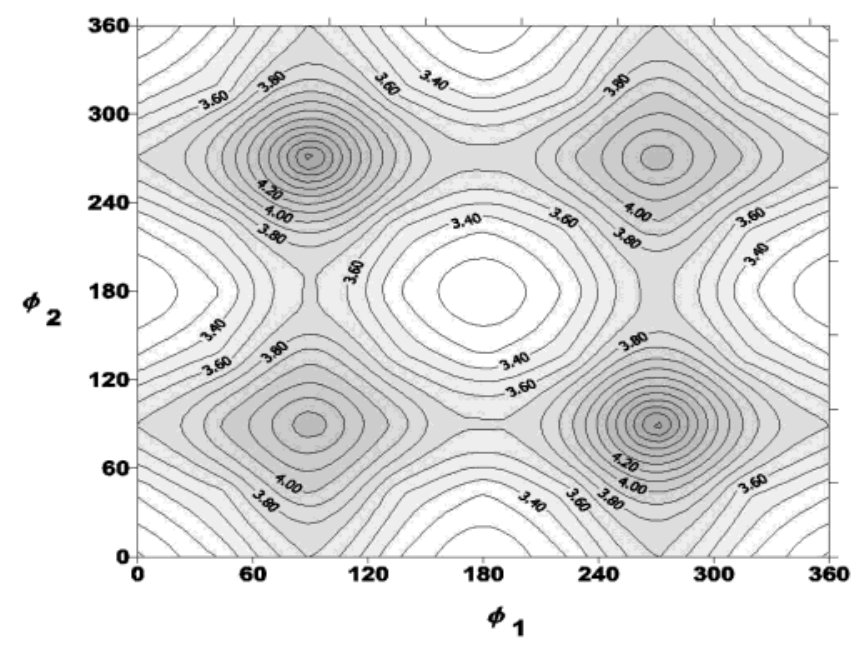

Figure 6. Excitation energy in methyl-3T $(\mathrm{eV})$.

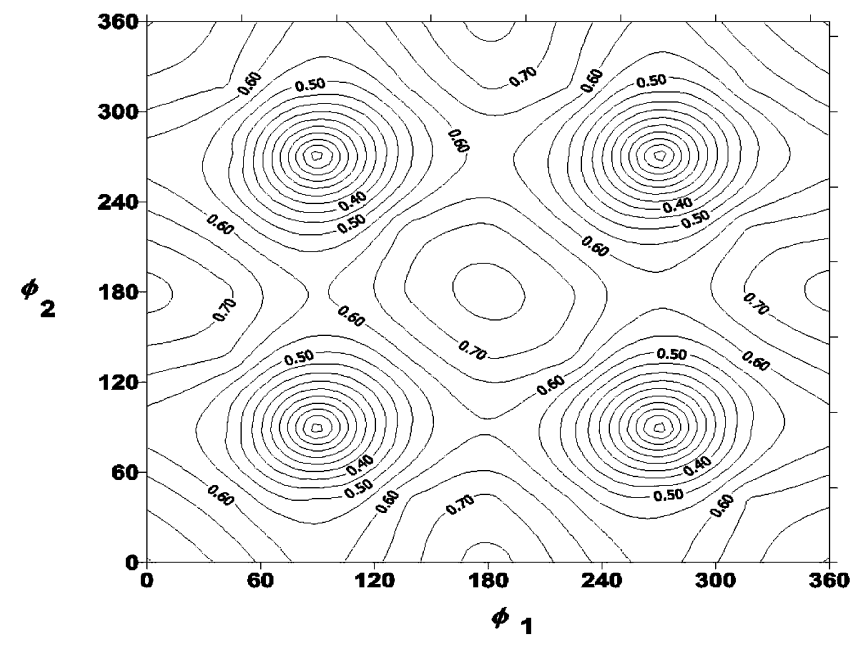

Figure 7. Intensity of absorption in methyl-3T (in au).

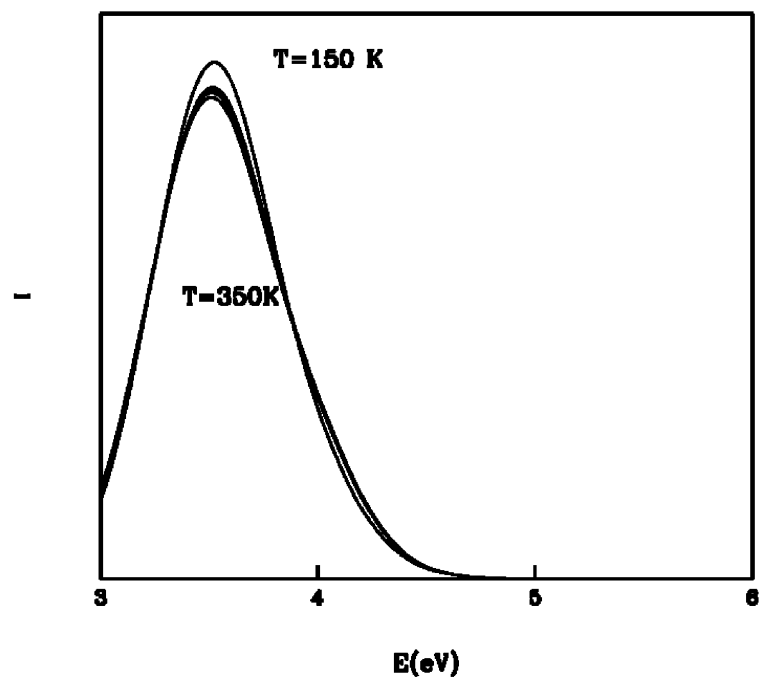

Figure 8. Temperature-dependent absorption spectra of methyl-3T.

\section{Conclusion}

The origin of the thermochromism in oligothiophenes has always been presented as the deviation from planarity with temperature. The basis of this belief comes from calculations showing a strong blue shift in the absorption peak as the torsional angle between two monomers changes from the minimum energy conformation. However, around room tem- perature the populations of the rotational isomers (at least in the gas phase) do not change significantly. Temperatures of the order of $1000-1500 \mathrm{~K}$ are required to populate higher energy states. We have reported extensive calculations for temperaturedependent UV-vis spectra of dimers and trimers of methylthiophene and we are not able to detect any traces of thermochromism. The longer alkyl groups are also seemed to be unlikely to have a different effect as the gas-phase rotational barriers are of the same magnitude. Oligomers in amorphous bulk should have even more difficulty in rotating from planar structures due to strong steric effects. This leaves us the only possible source of the thermochromism as the interaction between the oligomers and the surrounding neighbors. It is still possible that coupling between different oligomers are highly affected by the distribution of the torsional angles in the bulk; however, until this mechanism is clarified it is not correct to explain thermochromism with the rotation around the monomermonomer bonds alone.

\section{References and Notes}

(1) Leclerc, M.; Fiad, K. In Handbook of Conducting Polymers, 2nd ed.; Skotheim, T. A., Elsenbaumer, R. L., Reynolds, J. R., Eds.; Marcel Dekker: New York, 1998; pp 695-706.

(2) Nalwa, H. S. Ed. Handbook of Organic Conductive Molecules and Polymers; John Wiley: Chichester, 1997.

(3) Müllen, K., Wegner, G., Eds.; Electronic Materials; The Oligomer Approach; Wiley-VCH: Weinheim, 1998.

(4) Di Cesare, N.; Belletete, M.; Durocher, G.; Leclerc, M. Chem. Phys. Lett. 1997, 275, 533.

(5) Raymond, F. R.; Di Cesare, N.; Belletete, M.; Durocher, G.; Leclerc, M. Adv. Mater. 1998, 10, 599.

(6) Di Cesare, N.; Belletete, M.; Leclerc, M.; Durocher, G. Synth. Met. 1998, 94, 291

(7) Leclerc, M.; Dufresne, G.; Blondin, B.; Bouchard, J.; Belletete, M.; Durocher, G. Synth. Met. 2001, 119, 45 .

(8) Oka, K.; Fujiue, N.; Nakanishi, S.; Takata, T.; West, R.; Dohmaru, T. J. Organomet. Chem. 2000, 611, 45.

(9) Sanji, T.; Sakamoto, K.; Sakurai, H.; Ono, K. Macromolecules 1999, 32, 3788 .

(10) Salaneck, W. R.; Inganas, O.; Nilsson, J. O.; Österholm, J. E.; Themas, B.; Bredas, J. L. Synth. Met. 1989, 28, 451.

(11) Levesque, I.; Leclerc, M. Chem. Mater. 1996, 8, 2843.

(12) Yang, C.; Orfino, F. P.; Holdcroft, S. Macromolecules 1996, 29 , 6510

(13) Zerbi, G.; Chierichetti, B.; Inganas, O. J. Chem. Phys. 1991, 94, 4646.

(14) DiCesare, N.; Belletete, M.; Marrano, C.; Leclerc, M.; Durocher, G. J. Phys. Chem. A 1998, 102, 5142.

(15) Bagci-Kilic, G.; Toppare, L.; Yurtsever, E. Synth. Met. 1996, 78, 19.

(16) Di Cesare, N.; Belletete, M.;Marrano, C.; Leclerc, M.; Durocher, G. J. Phys. Chem. A 1999, 103, 795.

(17) Di Cesare, N.; Belletete, M.; Leclerc, M.; Durocher, G. J. Phys. Chem. A 1999, 103, 803.

(18) Di Cesare, N.; Belletete, M.; Garcia, E. R.; Leclerc, M.; Durocher, G. J. Phys. Chem. A 1999, 103, 3864.

(19) Yamamoto, T.; Komarudin, D.; Arai, M.; Lee, B.L.; Saganuma, H.; Asakawa, N.; Inoue, Y.; Kubota, K.; Sasaki, S.; Fukuda, T.; Matsuda, H. J. Am. Chem. Soc. 1998, 120, 2047.

(20) Tachibana, H.; Hosaka, N.; Tokura, Y. Macromolecules 2001, 34 1823

(21) Corish, J.; Morton-Blake, D. A.; Feeley, D. E.; Beniere, F.; Marchetti, M. J. Chem. Phys. B 1997, 101, 10075.

(22) Corish, J.; Morton-Blake, D. A.; Beniere, F.; Lantoine, M.; Marchetti, M. J. Mater. Sci. Forum 1997, 185, 239.

(23) Xie, H.; Corish, J.; Morton-Blake, D. A. Synth. Met. 2000, 113, 65 .

(24) Qiao, X.; Wang, X.; Mo, Z. Synth. Met. 2001, 118, 89

(25) Xie, H.; O’Dwyer, S.; Corish, J.; Morton-Blake, D. A. Synth. Met. 2001, 122, 287.

(26) Frisch, M. J.; Trucks, G. W.; Schlegel, H. B.; Scuseria, G. E.; Robb, M. A.; Cheeseman, J. R.; Zakrzewski, V. G.; Montgomery, J. A., Jr.; Stratmann, R. E.; Burant, J. C.; Dapprich, S.; Millam, J. M.; Daniels, A. D.; Kudin, K. N.; Strain, M. C.; Farkas, O.; Tomasi, J.; Barone, V.; Cossi, M.; Cammi, R.; Mennucci, B.; Pomelli, C.; Adamo, C.; Clifford, S.; Ochterski, J.; Petersson, G. A.; Ayala, P. Y.; Cui, Q.; Morokuma, K.; Malick, D. K.; Rabuck, A. D.; Raghavachari, K.; Foresman, J. B.; Cioslowski, J.; 
Ortiz, J. V.; Stefanov, B. B.; Liu, G.; Liashenko, A.; Piskorz, P.; Komaromi, I.; Gomperts, R.; Martin, R. L.; Fox, D. J.; Keith, T.; Al-Laham, M. A Peng, C. Y.; Nanayakkara, A.; Gonzalez, C.; Challacombe, M.; Gill, P. M. W.; Johnson, B. G.; Chen, W.; Wong, M. W.; Andres, J. L.; Head-Gordon, M.; Replogle, E. S.; Pople, J. A. Gaussian 98; Gaussian, Inc.: Pittsburgh, PA, 1998.

(27) Becke, A. D. J. Chem. Phys. 1996, 104, 1040

(28) Karpfen, A.; Choi, C. H.; Kertsez, M. J. Phys. Chem A 1997, 101, 7426.
(29) Bongini, A.; Bottoni, A. J. Phys. Chem. A 1999, 103, 6800.

(30) Casida, M. E. Recent Advances in Density Functional Theory; Chong, D. P., Ed.; World Scientific: Singapore, 1999; p 100.

(31) Della Sala, F.; Heinze, H. H.; Görling, A. Chem. Phys. Lett. 2001, 339, 343.

(32) Pearl, G. M.; Zerner, M. C.; Broo, A.; McKelvey, J. J. Comput. Chem. 1998, 19, 781.

(33) Yurtsever, M.; Yurtsever, E. Synth. Met. 2001, 119, 247. 